\title{
THE REEL ALLOCATION PROBLEM OF A CORRUGATED PACKAGING COMPANY
}

\author{
Marcelo Ferreira $^{1}$, Victor Claudio Bento Camargo ${ }^{2}$ \\ and Silvio Alexandre de Araujo ${ }^{3 *}$
}

Received August 10, 2019 / Accepted April 09, 2020

\begin{abstract}
In this paper we address a reel allocation problem that appears in a corrugated cardboard packaging company. Given a production plan which incorporates customer orders and a set of reels in stock, the decision is how to allocate these reels to the corrugator to produce the ordered corrugated cardboard sheets, aiming to minimize the number of reels used, any partial consumption of reels, the unusable leftovers and the number of production stoppages. A mixed-integer mathematical model is proposed and computational tests were performed using instances generated based on real data. The computational results show that the proposed mathematical model is consistent with the reality of the studied company and brings interesting insights that can be used in the decision-making.
\end{abstract}

Keywords: mixed-integer programming, mathematical modeling, corrugated packaging industry, reel allocation problem, corrugator.

\section{INTRODUCTION}

Reusable and recyclable packaging is widely used nowadays because it reduces costs, complies with environmental laws, optimizes the use of space and does not pose any risk to health or contaminate products. In this context, corrugated cardboard packaging stands out, given its versatility to serve the most varied markets. The most common corrugated cardboard is composed of three layers of sheets: the outer cover, the core and the inner cover.

Corrugated cardboard packaging companies (CCPC) are immersed in a modern, highly competitive and globalized market and are increasingly in need of organization and strategy to be successful. According to Darley et al. (2004), flexibility is fundamental in dealing with ever-

\footnotetext{
*Corresponding author

${ }^{1}$ Universidade Federal do Triângulo Mineiro-UFTM, Av. Dr. Randolfo Borges Júnior, 1400, Univerdecidade, Uberaba, MG, Brasil - E-mail: marcelo.ferreira@uftm.edu.br - https://orcid.org/0000-0002-6854-1837

${ }^{2}$ Universidade Federal de São Carlos (UFSCar), Rodovia Washington Luis, Km 235, CEP 13565-905 São Carlos, SP, Brasil - E-mail: victor.camargo@dep.ufscar.br - https://orcid.org/0000-0001-9332-3025

${ }^{3}$ Universidade Estadual Paulista Júlio de Mesquita Filho-UNESP, Rua Cristovão Colombo, 2265, Jardim Nazareth, São José do Rio Preto, SP, Brasil - E-mail: silvio.araujo@unesp.br - https://orcid.org/0000-0002-4762-2048
} 
increasing demand, the wide variety of orders and multi-stage production processes. One way to improve flexibility is to solve the problem of production planning.

Most production planning research in a CCPC has addressed the problem of determining cutting patterns Haessler \& Talbot (1983). There are few studies that look at other aspects such as the planned use of reels in the corrugator (multi-stage machine which produces the rectangles that will give rise to the cardboard packaging). This planning is necessary therefore, when defining how much will be produced from each type of cardboard (planning schedules), it is necessary to decide which reels will be used in the corrugator at minimal cost.

Following a technical visit to a CCPC, this study looks at the relevance of the costs related to the allocation of reels to the corrugator and a mathematical model that considers various aspects of this planning is proposed to help in the decision-making. In addition, a set of instances is generated based on real data and computational results are presented proving the relevance of the proposed model.

The paper is organized as follows. A literature review in Section 2 shows that few papers address this type of problem. In Section 3, there is a description of the production process in a CCPC and a detailed description of the problem being investigated. In Section 4, a mixed-integer mathematical model is presented, as well as an illustrative example. Section 5 contains the computational results and Section 6 the conclusions and ideas for future work.

\section{LITERATURE REVIEW}

Although the proposition of an optimization framework for the specific industrial application being considered in this paper is barely addressed, we will relate the problem to its context in the literature. So, this section presents some related literature, especially studies in the corrugated packaging and paper industries and to the cutting stock problems with leftovers.

We have found only three papers (Chantrapornchai \& Sathapanawat, 2010, 2011, 2012), all belonging to the same authors, that address aspects of programming the use of reels in the corrugator. The aim of these papers is to develop software to minimize the number of unusable leftovers and to minimize the leftovers on reels that had been partially used (which they denominated "stumps"). Secondary objectives are the minimization of the number of reels in each execution plan and the use of paper in the order of aging. A heuristic is presented in Chantrapornchai \& Sathapanawat (2011), with the objective of providing the sequence of use of the reels for each reel holder. In Chantrapornchai \& Sathapanawat (2012), a case study is presented, where the software deployed is tested with real examples. Another paper that is related to our current research is Matsumoto et al. (2009), where the main objectives are the minimization of lateral losses, the minimization of the length of paper on used reels and the minimization of the number of stoppages in the corrugator. To the best of our knowledge, this is the first and only study to consider the problem of minimizing stoppages.

Considering other research related to the corrugated packaging and paper industries but not directly related to the problem studied in this paper, Pegels (1967) compares the three heuristics 
for cutting programming in the context of the cardboard packaging industry. The author analyzes the results in terms of order break-up, waste and corrugator width utilization. In Bookbinder \& Higginson (1986), the trade-off between customer service (due dates) and trim waste is analyzed. In Lins (1989), the problem of sequencing the schedules in the corrugator is addressed with the objective of minimizing the open stacks. Savsar \& Cogun (1994) present a mathematical model to minimize lateral loss and it includes storage capacity restrictions. Bolat (2000) extends the paper of Savsar \& Cogun (1994) by considering the trade-off between total lateral loss and setups. A generalization of the ideas presented in Lins (1989) is considered in the paper by Becceneri et al. (2004), where a new heuristic is used to calculate an upper bound of the open stacks as well as an exact method is proposed. In Velasquez et al. (2007) an approach to the corrugator programming problem is proposed using a multi-objective evolutionary algorithm.

Rodríguez \& Vecchietti (2007) consider the cutting stock problem in a cardboard packaging factory. First, a mathematical model generates the cutting patterns, which will be used in the second stage, where a mixed integer linear programming model optimizes the cutting process, minimizing lateral losses. Poltroniere et al. (2008) propose an integrated mathematical model to represent the lot sizing problem in the production of large paper reels and the cutting stock problem to produce smaller reels. Two decomposition heuristics are also proposed to solve the model by approaching one problem at a time. In Rodríguez (2009), a combination of a greedy algorithm with a genetic algorithm is proposed to deal with the problem of minimizing lateral losses. This idea is also present in a paper by Jaramillo et al. (2009), who point out that methods based on genetic algorithms obtain better results than those based on linear programming. In Santos \& Almada-Lobo (2012), a real-world production planning and scheduling problem occurring at an integrated pulp and paper mill is considered. Due to significant sequence-dependent setups in paper type changeovers, sizing and sequencing of lots have to be made simultaneously. The benefits of integrating the stages are discussed. The proposed approaches are tested on real-world data. In Rodriguez \& Vecchietti (2013), the authors consider integrated decisions related to the cutting stock and scheduling problems with due dates applied in the corrugated cardboard packaging industry.

Finally, also relevant to this research are papers that considered the cutting stock problem with usable leftovers (Arenales et al., 2015; Cherri et al., 2014) where the leftovers can be used to cut future items and are not considered as waste, which is the same as the reels that are partially used in the corrugated industry. A review of the literature about this problem can be found in Cherri et al. (2014).

\section{PROBLEM DEFINITION}

Figure 1 illustrates, in simple terms, the flow of materials and the main decisions made in the CCPC production process. The following explanation will refer to Figure 1 by using its numbers. At the beginning of the process, the raw material in stock (1) is used for the paper production (2) and then is wrapped onto the reels. The reels are stored (3) for future use in the production of packaging. 
In planning the production of the corrugated packaging, schedules are defined. These schedules consist of a plan for packaging production which incorporates customer orders using a strategy defined by Production Planning and Control (PPC) department of the CCPC. A schedule defines the type of packaging ( 3 or 5 layers), the size of the sheet, its weight and which blanks must be produced. The blanks are rectangles cut from the sheets that give rise to the packaging. By knowing the size of the sheets, the required paper length in each layer is defined.

Once the schedule has been defined, it is possible to choose the reels in stock that will be used for the production of the corrugated sheets. The choice of reels (4) can be designed to increase the efficiency of the production processes and reduce the need for rework of previous processes.

The reels chosen from stock are installed in the corrugator (5) for the production of the sheets (6). The blanks are then cut from these sheets (7) and sent to intermediate stock (8), where they subsequently undergo additional trimming and printing of information (9), as required by customers. Some cardboard packaging, depending on their specifications, may go through other steps, such as stapling or sticking with special glue. At this point the cardboard packages are packed (10) and stored (11), available for future distribution.

Figure 1 - Flowchart of the corrugated packaging production process.

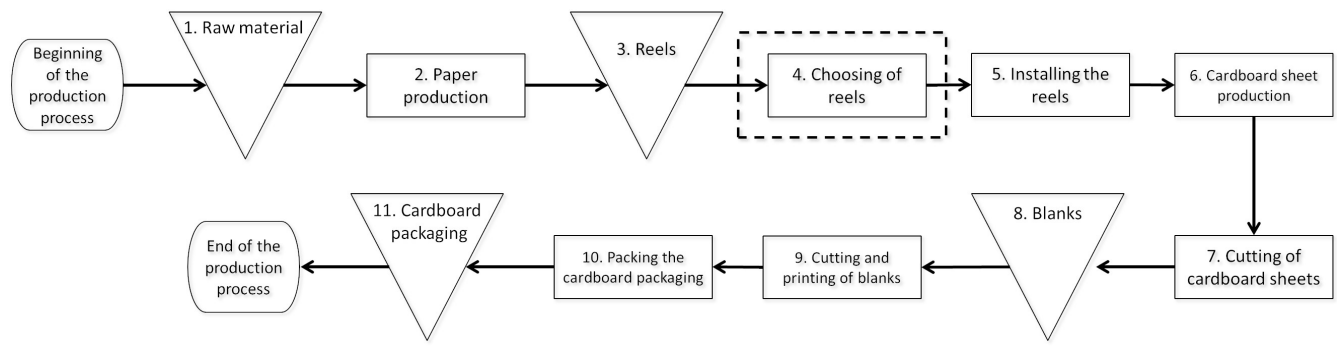

The Reel Allocation Problem refers to the planning decisions involved in Procedure (4) as highlighted in the Figure 1. These decisions aim to choose which reels should be allocated to the corrugator. Figure 2 illustrates a corrugator producing a cardboard sheet of three layers. Note that for each layer a reel holder assembly is required. The reels are installed on the reel holders in pairs, keeping one on hold for use when the first reel is completely used or needs to be replaced due to a production strategy. Three sets of reels are needed for single wall corrugated board and five sets for double wall corrugated board.

\subsection{Aspects of the production process}

Replacement of reels in the corrugator reel holder assemblies is quite frequent and can be caused by one of the following: i) the length of the initially installed reels is not sufficient to produce the sheet length described in the schedule; ii) a reel is damaged during the production process or iii) because the subsequent schedule needs a different weight. When producing a sheet of corrugated packaging, a reel may have leftover paper in the reel holders. A reel not used in its entirety may 
Figure 2 - Illustration of reel holder assemblies for a 3 layer sheet.

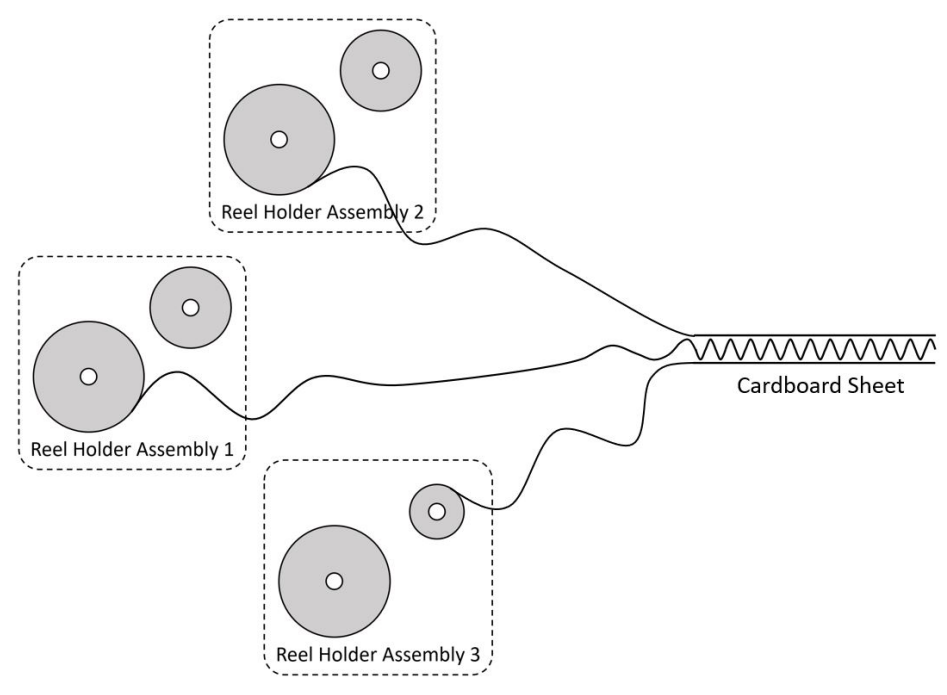

be re-usable or not. If it is usable, the partially-used reel returns to stock and is used in a new schedule of equal or lower weight; if it is not usable, it is sent for recycling. An unusable leftover is a partially-used reel of undesirable length, as previously defined by the CCPC.

Another important aspect of the production process is related to the use of reels, which can be partially used or fully used. In practice, the use of a full reel has priority over partial use. With partial use, the corrugator needs programming to measure the usage and its manual interruption by the operator. In general, partial usage is done by approximation, that is, part of the reel is used leaving a leftover that the corrugator operator considers acceptable. For partial use, a minimum use is established. In relation to cost, partial use is more expensive because, after use, the reel must be transported back to stock, which does not occur when the whole reel is used. Also, there is the extra cost of managing the stock of partially-used reels.

Another point of great relevance to this planning is obtaining solutions that minimize stoppages of the corrugator as this causes productivity losses. The stoppages we refer to here are those caused by bad choices of reels and not by unpredictable factors such as mechanical failures or tears. A reel installed in a reel holder assembly must be of a minimum length to last the time needed to change its paired reel, thus preventing the corrugator from stopping at the time of its replacement. This length depends on the operating speed of the corrugator. In practice, stoppages are avoided by using large reels during the production process as they allow for a large number of replacements of the reel paired to it. However, the frequent use of large reels causes the accumulation of partially used reels in stock. In addition, due to the complexity of manually including the short length reels in the production of the same schedule, there is a need to acquire or produce new reels, which implies poor financial management. 
This planning problem leads to a non-trivial analysis of which reels will be used to minimize the number of corrugator stoppages. Related to this problem, a sequencing problem also arises. After choosing the reels that will be allocated to the corrugator, defining how much paper from each reel will be used and which reel holder they will be installed on, the order in which they will be used needs to be defined. Since each reel holder is composed of two support pins, the sequence in which the reels will be used is fundamental to the corrugator not being interrupted. This sequencing problem is not considered in this paper and must be solved by the Production Planning and Control (PPC) department of the CCPC. A procedure that simulates the practice is described in Appendix.

Finally, in order to reduce the costs associated with the allocation of reels in the corrugator, minimizing the total number of reels used in a schedule is relevant to the context of the CCPC production process.

Summarizing, the reel allocation problem assists a CCPC decision maker in defining which reels will be used for the production of a cardboard sheet ordered by the PPC department. The chosen set of reels should have minimum costs of transporting the reels between the stock and the corrugator, installing the reels on the pins, recycling the paper from the unusable reels and stopping the corrugator. An example of the application of this problem and a discussion of the conflicts among the objectives are found in Section 4.1.

\section{MATHEMATICAL MODELING FOR THE REEL ALLOCATION PROBLEM}

Considering that the problem of defining schedules is solved a priori by the PPC, the reel allocation problem previously described can be solved by applying the mathematical model proposed in this section. The decision involves determining which reels to allocate in the corrugator in order to produce the demanded corrugated cardboard sheets, aiming to minimize costs related to the number of reels used, the partial consumption of reels, the unusable leftovers and the number of production stoppages.

Consider the following indices, parameters and decision variables of the reel allocation problem:

\section{Indices:}

- $i=1 \ldots m$, where $m$ represents the number of reels.

- $j=1 \ldots n$, where $n$ represents the total number of layers of sheets to be produced.

\section{Parameters:}

- $w_{i}$ : Length of paper on reel $i$.

- $c_{j}$ : Length of layer $j$.

- $p$ : Minimum length for a reel to last the time needed to exchange its paired reel.

- $s$ : Minimum length for a reel to be usable. 
- $v$ : Minimum length of paper on a reel to be used in case of partial usage.

- $\beta_{1}$ : Cost of transporting a reel to the corrugator and of putting the reel on the appropriate pin.

- $\beta_{2}$ : Cost of transporting a reel from the corrugator back to stock.

- $\beta_{3}$ : Cost of recycling one meter of unusable paper.

- $\beta_{4}$ : Cost of the corrugator being stopped.

\section{Decision variables:}

- $Y_{i j}$ : Assumed to be 1 if reel $i$ is allocated to layer $j$ and 0 otherwise.

- $X_{i j}$ : Length of paper from reel $i$ used in layer $j\left(0 \leq X_{i j} \leq w_{i}\right)$.

- $T_{i j}$ : Assumed to be 1 if reel $i$ allocated to layer $j$ is partially used and 0 otherwise (when the reel is fully used or not used at all in layer $j$ ).

- $P_{j}$ : Number of stoppages caused by the set of reels producing layer $j$.

- $Z_{i j}$ : Number of changes that can be made in layer $j$ when reel $i$ is used in layer $j$.

- $A_{i j}$ : Assumed to be 1 when reel $i$ is allocated to layer $j$ and the unused paper is unusable and 0 otherwise, i.e., when the reel does not generate waste paper or the reel is not used at all.

- $D_{i j}$ : Length of unusable paper left on reel $i$ used in layer $j$. Assumed to be 0 when the reel $i$ is fully used, partially used but re-usable or the reel is not used at all.

Also, consider the following observations about reel changes, possible reel changes and corrugator stoppages.

Definition 4.1. A reel change occurs in the assembly when one reel replaces another reel in the same schedule. The first two reels placed in each reel holder are not considered exchanges as they do not replace any scheduled reel to be processed. Thus, the amount of changes needed for a set with $m$ reels allocated to reel holder $j$ is equal to $\left(\sum_{i=1}^{m} Y_{i j}\right)-2$.

Definition 4.2. The number of changes supported by a reel (during the time that paper is being used from it) is equal to the number of reels paired to it that can be replaced without stopping. Thus, the number of changes supported by a set with $m$ reels allocated for the reel holder $j$ is given by $\sum_{i=1}^{m}\left\lfloor X_{i j} / p\right\rfloor$.

Definition 4.3. A stoppage occurs when the number of reel changes supported by a set with $m$ reels is less than the number of changes needed for this same set of reels. Let $P_{j}$ be the number of stoppages for a set with $m$ reels, then $P_{j} \geq\left(\sum_{i=1}^{m} Y_{i j}\right)-2-\sum_{i=1}^{m}\left\lfloor X_{i j} / p\right\rfloor$. 
In fact, if $\left(\sum_{i=1}^{m} Y_{i j}\right)-2 \leq \sum_{i=1}^{m}\left\lfloor X_{i j} / p\right\rfloor$, then $P_{j} \geq 0$, it follows that $P_{j}=0$ (since the aim is to minimize the number of stoppages), that is, there will be no stoppages. However, if $\left(\sum_{i=1}^{m} Y_{i j}\right)-$ $2>\sum_{i=1}^{m}\left\lfloor X_{i j} / p\right\rfloor$, then $P_{j}=\left(\sum_{i=1}^{m} Y_{i j}\right)-2-\sum_{i=1}^{m}\left\lfloor X_{i j} / p\right\rfloor>0$, because the aim is to minimize $P_{j}$. This difference is exactly the number of stoppages for each reel holder $j$. It should be noted that the number of stoppages per reel holder is equivalent to the number of stoppages per layer since each reel holder only provides paper for one layer of the sheet.

\section{Model of the Reel Allocation Problem:}

$$
\operatorname{Minimize} \sum_{i=1}^{m} \sum_{j=1}^{n} \beta_{1} \cdot Y_{i j}+\sum_{i=1}^{m} \sum_{j=1}^{n} \beta_{2} \cdot T_{i j}+\sum_{i=1}^{m} \sum_{j=1}^{n} \beta_{3} \cdot D_{i j}+\sum_{j=1}^{n} \beta_{4} \cdot P_{j}
$$

\section{Subject to:}

$$
\begin{array}{lr}
\sum_{j=1}^{n} Y_{i j} \leq 1 ; & \forall i \\
X_{i j} \leq w_{i} \cdot Y_{i j} ; & \forall i, j \\
\sum_{i=1}^{m} X_{i j}=c_{j} ; & \forall j \\
Z_{i j} \leq X_{i j} / p ; & \forall j \\
Z_{i j} \geq\left(X_{i j} / p\right)-1 ; & \forall j \\
P_{j} \geq \sum_{i=1}^{m}\left(Y_{i j}\right)-2-\sum_{i=1}^{m}\left(Z_{i j}\right) ; & \forall j \\
w_{i} \cdot Y_{i j}-X_{i j} \geq s \cdot\left(1-A_{i j}\right) ; & \forall i, j \\
w_{i} \cdot Y_{i j}-X_{i j} \leq w_{i} \cdot\left(1-A_{i j}\right)+s \cdot A_{i j} ; & \forall i, j \\
D_{i j} \leq s \cdot A_{i j} ; & \forall i, j \\
D_{i j} \geq w_{i} \cdot Y_{i j}-X_{i j}-\left(1-A_{i j}\right) \cdot w_{i} ; & \forall i, j \\
X_{i j} \geq v \cdot T_{i j} ; & \forall i, j \\
w_{i} \cdot Y_{i j}-X_{i j} \leq w_{i} \cdot T_{i j} ; & \forall i, j \\
Y_{i j}, T_{i j}, A_{i j} \in\{0,1\}, Z_{i j}, P_{j} \in \mathbb{Z}^{+}, X_{i j}, D_{i j} \in \mathbb{R}^{+} &
\end{array}
$$

The objective function (1) aims to minimize allocation costs, partial usage costs, unused paper recycling costs and stoppage costs.

Constraints (2) ensure that each reel is assigned at most once in some reel holder assembly. Constraints (3) determine that paper is only used from a reel assigned to the respective reel holder assembly. In addition, the consumption of the reel is limited to its length. Constraints (4) ensure that the paper length used from the reels in each layer meets the demand of the schedule. Constraints (5) and (6) linearize the equation $Z_{i j}=\left\lfloor X_{i j} / p\right\rfloor$ that define the number of changes 
supported by the reel $i$ allocated to the reel holder $j$. Constraints (7) deal with the number of stoppages for a given reel holder assembly.

Constraints (8) and (9) classify a reel after its use $\left(w_{i} \cdot Y_{i j}-X_{i j}\right)$ as usable or not usable. If $w_{i} \cdot Y_{i j}-X_{i j}>s$, then $A_{i j}=0$ and the leftover is defined as usable. If $w_{i} \cdot Y_{i j}-X_{i j}<s$, then $A_{i j}=1$ and the remainder is defined as unusable. However if $w_{i} \cdot Y_{i j}-X_{i j}=s$, by constraints (8) and (9), $A_{i j}=0$ or $A_{i j}=1$. This lack of definition is resolved by Constraints (10) and (11). Constraints (10) and (11) define the length $D_{i j}$ of an unusable leftover, i.e. when $A_{i j}=1$. In this case, $w_{i}$. $Y_{i j}-X_{i j} \leq D_{i j} \leq s$. As $D_{i j}$ is present in the minimization objective function, $D_{i j}=w_{i} \cdot Y_{i j}-X_{i j}$. When $A_{i j}=0$, then $D_{i j}=0$, because $D_{i j} \in \mathbb{R}^{+}$. Similarly, the undefined case $w_{i} \cdot Y_{i j}-X_{i j}=s$ is solved by minimizing $D_{i j}$, fixing the leftover as usable, $A_{i j}=0$ and $D_{i j}=0$.

Constraints (12) determine if the partial use is greater than the predefined value. Constraints (13) define the length of the leftover paper when there is partial use of the paper on the reel. If the reel $j$ is not used at all or is fully used, $T_{i j}=0$. In (14) the domains of the variables are given.

\subsection{Illustrative example}

Figures 3 to 7 show illustrations of 5 feasible solutions for the same problem instance of the reel allocation problem. The gray rectangles represent the length of paper required to produce each layer. The white rectangles indicate the reels used and their lengths. Consider that a cardboard sheet of $n=3$ layers should be produced where the inner and outer covers need $c_{1}=c_{3}=2000$ meters of paper and the core needs $c_{2}=3000$ meters. There are $m=19$ reels in stock with the following paper lengths $w=$ $\{100,150,200,250,300,350,400,450,500,550,600,650,700,750,800,850,900,950,1000\}$

- in meters. In this illustrative example, the minimum paper length used from a reel in case of partial use is $v=100 \mathrm{~m}$. The minimum paper length for a reel to be considered usable is $s=100 \mathrm{~m}$. And, the minimum paper length for a reel to allow changing to its paired reel is $p=300 \mathrm{~m}$. The costs are $\beta_{1}=5.11, \beta_{2}=4.35, \beta_{3}=0.05$ and $\beta_{4}=480.42$ currency units. Observe that, in this example, we are considering only the reel allocation problem, i.e., the sequencing problem is not considered.

Solution $S^{1}$ was built by allocating the largest reel available in stock to the reel holder assembly that had the smallest amount of paper allocated. The reels are allocated until the amount of paper needed for each layer is satisfied. As can be seen in this solution in Figure 3, in each of the three layers, one reel is partially used. The leftovers are returned to stock because the amount of paper in each one is greater than the minimum value $s$ for the reel to be considered usable. There are no stoppages in this solution.

Solution $S^{2}$ was built by allocating to a reel holder assembly the smallest reels available in stock until the amount of paper needed for each layer is satisfied. There is partial consumption of reels in the outer and inner covers with the leftovers returning to stock as can be seen in Figure 4. There are two stoppages in the outer cover that can be calculated using the constraints (7) of the mathematical model. 
Figure 3 - Illustration of a feasible solution. Solution $S^{1}$.

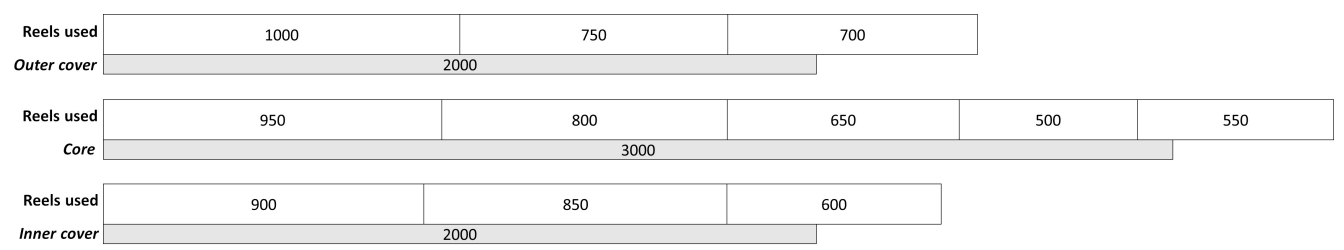

Figure 4 - Illustration of a feasible solution. Solution $S^{2}$.

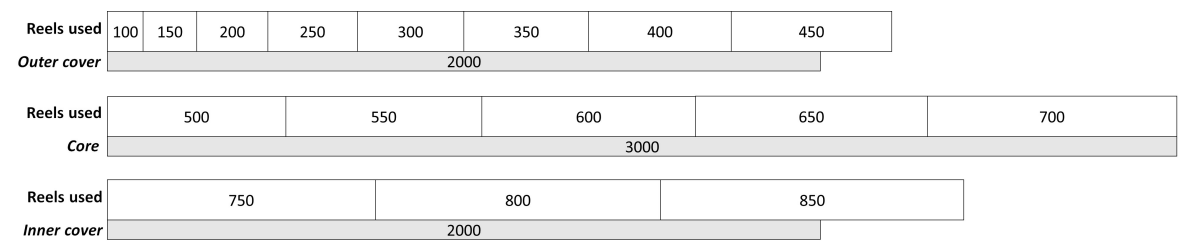

Solution $S^{3}$ was designed by alternately allocating to a reel holder assembly, the largest reel and the smallest reel available in stock until the amount of paper required for each layer is satisfied. As illustrated in Figure 5, there is partial consumption of reels in the inner cover and in the core with the leftovers returning to stock. On the outer cover, the leftover is considered unusable as the amount of paper on the reel is less than the minimum value for the leftover on the reel to be considered usable. There are no stoppages.

Figure 5 - Illustration of a feasible solution. Solution $S^{3}$.

\begin{tabular}{|c|c|c|c|c|c|c|}
\hline \multirow{2}{*}{$\begin{array}{l}\text { Reels used } \\
\text { Outer cover }\end{array}$} & 1000 & 100 & \multicolumn{2}{|c|}{950} & & \\
\hline & \multicolumn{4}{|c|}{2000} & & \\
\hline \multirow{2}{*}{$\begin{array}{r}\text { Reels used } \\
\text { Core }\end{array}$} & 900 & 150 & 850 & 200 & 800 & 250 \\
\hline & \multicolumn{4}{|c|}{3000} & & \\
\hline \multirow{2}{*}{$\begin{array}{l}\text { Reels used } \\
\text { Inner cover }\end{array}$} & 750 & & 700 & 350 & & \\
\hline & \multicolumn{4}{|c|}{2000} & & \\
\hline
\end{tabular}

Solutions $S^{4}$ and $S^{5 *}$ represent cases where there are no partially used reels and consequently no leftovers. The chosen reels exactly meet the paper requirements in all layers. The difference between the solutions lies in the number of reels chosen and in the number of stoppages. Solution $S^{4}$ uses 14 reels and solution $S^{5 *}$ uses 10 reels as can be seen in Figures 6 and 7 respectively. Besides, solution $S^{5 *}$ is optimal.

Table 1 presents a comparison among the feasible solutions for the illustrative example. The following data are presented: $\sum Y$ - number of used reels; $\sum T$ - number of reels that return to stock as they are usable leftovers; $\sum D$ - amount (in meters) of unusable paper that is sent to recycling; $\sum P$ - number of corrugator stoppages; OF - and the value of the objective function. 
Figure 6 - Illustration of a feasible solution. Solution $S^{4}$.

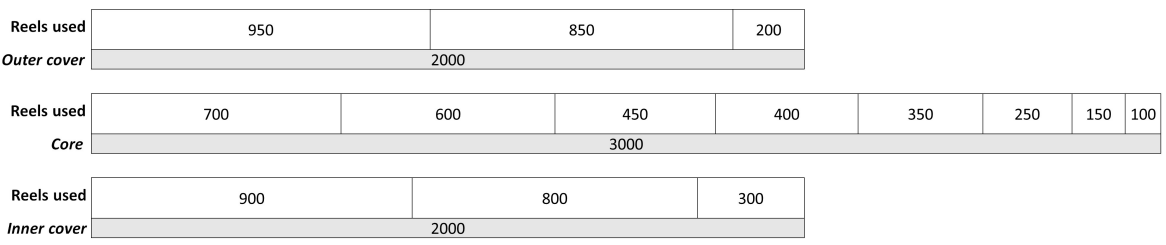

Figure 7 - Illustration of a feasible solution. Solution $S^{5 *}$.

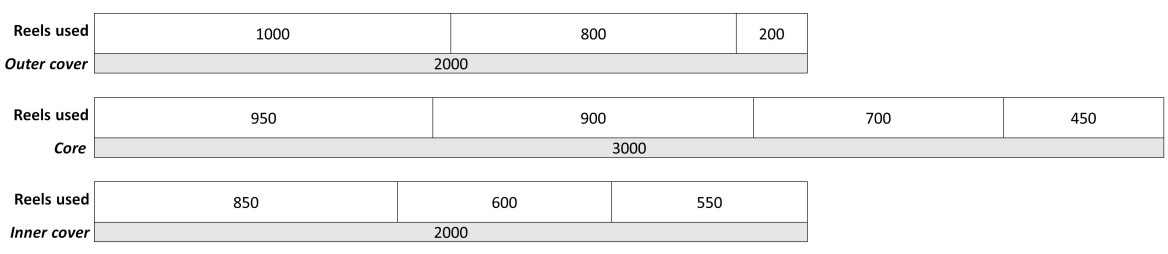

Table 1 - Comparison among the objective terms of the solutions.

\begin{tabular}{cccccr}
\hline & $\sum Y$ & $\sum T$ & $\sum D$ & $\sum P$ & OF \\
\hline$S^{1}$ & 11 & 3 & 0 & 0 & 56.21 \\
$S^{2}$ & 16 & 2 & 0 & 2 & 1051.30 \\
$S^{3}$ & 13 & 3 & 50 & 0 & 81.98 \\
$S^{4}$ & 14 & 0 & 0 & 0 & 71.54 \\
$S^{5}$ & 10 & 0 & 0 & 0 & 51.10 \\
\hline
\end{tabular}

By considering the set of feasible solutions for the illustrative example and the mathematical model (1)-(14), some insights from the conflicts of objectives can be drawn: the higher the number of reels used $\left(\sum Y\right)$, the higher the chance of a stoppage in the corrugator affecting $\beta_{4}$ costs. On the other hand, the smaller the number of reels used, the more difficult it is to combine the reels to meet paper needs with accuracy. This case may imply usable and unusable leftovers implying increased costs $\beta_{2}$ and $\beta_{3}$; the increase in the number of partially used reels $\left(\sum T\right)$ directly affects the total number of reels used and $\beta_{1}$ costs; the increase in the quantity of paper considered unusable $\left(\sum D\right)$ may mean an increase in the total number of reels used and in $\beta_{1}$ costs.

\section{COMPUTATIONAL RESULTS}

In order to validate the model, in this section, computational results are presented based on real data from a CCPC. Initially, a description of how the data are generated and then the general computational results are presented as well as an analysis of the influ- 
ence of each parameter on the results. The generated instances are available on GitHub (https://github.com/VictorCBCamargo/Corrugated).

All computational tests were performed using a computer with an Intel Core i7-2600 processor, 3.4 GHz CPU and 16 GB RAM. The mathematical model was coded in AMPL and solved with CPLEX 12.7. The processing time for each instance has been fixed at 1800 seconds, as it is an acceptable time in practice. In some instances this time limit was exceeded for a few seconds due to the read and write times of the data.

\subsection{Parameters defined by company policy}

The input parameters of the mathematical model are detailed in Section 4 and can be divided into two groups: the parameters defined by company policy and the parameters that are schedule and stock dependent. Schedule and stock dependent parameters are distinct for each instance of the problem and are described in Section 5.2. The parameters defined by company policy and described next are in line with the practice.

$v$ : The minimum paper length used from a reel in case of partial use is $v=450 \mathrm{~m}$.

$s$ : The minimum paper length for a reel to be considered usable is $s=300 \mathrm{~m}$.

$p$ : The minimum paper length for a reel to allow changing to its paired reel is $p=750 \mathrm{~m}$.

$\beta_{1}$ : Cost of using a reel, includes transport to the corrugator and the preparation of the reel (includes placing and removing the reel from the reel holder). It is derived from the following costs: forklift operator wages, the cost of renting the forklift and the salary of the corrugator operators. Using real data, we get $\beta_{1}=5.11$ currency units.

$\beta_{2}$ : Cost of partial consumption. In addition to the cost of using a reel, the cost of transporting the corrugator reel back to stock is taken into account. $\beta_{2}=4.35$ currency units.

$\beta_{3}$ : Cost of recycling one meter of unused paper, $\beta_{3}=0.05$ currency units.

$\beta_{4}$ : Cost associated with a corrugator stoppage (one minute), $\beta_{4}=480.42$ currency units. All stoppages are assumed to last for one minute.

\subsection{Schedule and stock dependent parameters}

As stated earlier, the schedule and stock dependent parameters are different for each instance, which allows the characterization of the instances used in this work.

Instances take into consideration the length of the schedule, the type of paper needed, and the available stock of reels. An instance generator was used to build a set of different situations in the production environment, considering different configurations of schedules and stocks of reels.

An instance class is characterized by four aspects - number of layers in the schedule, number of reels in stock, paper lengths on reels in stock and schedule lengths. The following parameters were considered: 
$n$ : Number of schedule layers. It can be single wall (S) with 3 layers or double wall (D) with 5 layers.

$m$ : Number of reels in stock. Can be small (S) with 100 reels, medium (M) with 200 reels and large (L) with 300 reels.

$w_{i}$ : Lengths of reels in stock. Values are random numbers according to the scenarios described in Table 2.

Table 2 - Parameters according to the paper lengths on reels in stock.

\begin{tabular}{lccc}
\hline & \multicolumn{3}{c}{ Paper lengths on reels } \\
\hline Stock profile & $300 \mathrm{~m}-749 \mathrm{~m}$ & $750 \mathrm{~m}-6499 \mathrm{~m}$ & $6500 \mathrm{~m}$ \\
\hline Small $(\mathbf{S})$ & $90 \%$ & $5 \%$ & $5 \%$ \\
Medium $(\mathbf{M})$ & $80 \%$ & $10 \%$ & $10 \%$ \\
Large $(\mathbf{L})$ & $70 \%$ & $15 \%$ & $15 \%$ \\
\hline
\end{tabular}

$c_{j}$ : Total length of the schedule. A small schedule (S) consumes $65 \%$ of the total length. of reels in stock. A medium schedule (M) consumes $80 \%$ and a large schedule (L) consumes $95 \%$ of the total length of reels in stock.

Instance classes are named after their four characteristics. For example, class SSML contains instances with a Simple layer, a Small number of reels in stock, Medium paper lengths on reels in stock and a Large length schedule. For each of the 54 classes, 10 instances were generated, by varying the lengths of the reels in stock (see Table 2), totaling 540 instances.

\subsection{General analysis of results}

Table 3 gives the overall results for the 540 instances and Table 4 gives the average of the values obtained over the 10 instances in the 54 classes tested.

Table 3 - General analysis of the 540 instances.

\begin{tabular}{lcc}
\hline Item analyzed & Totals & Percentages \\
\hline Total instances analyzed & 540 & $100 \%$ \\
\hline Instances with proof of optimality & 266 & $49.25 \%$ \\
Instances with no proof of optimality & 274 & $50.75 \%$ \\
\hline Instances with unusable reels & 36 & $6.67 \%$ \\
Instances with stoppages & 60 & $11.11 \%$ \\
\hline Instances with all reels totally used & 341 & $63.14 \%$ \\
Instances with one partially used reel & 63 & $11.66 \%$ \\
Instances with two partially used reels & 45 & $8.33 \%$ \\
Instances with three partially used reels & 44 & $8.14 \%$ \\
Instances with four partially used reels & 24 & $4.44 \%$ \\
Instances with five partially used reels & 23 & $4.25 \%$ \\
\hline
\end{tabular}


Table 4 - Average results of the 10 instances of each class.

\begin{tabular}{|c|c|c|c|c|c|c|c|c|c|c|}
\hline & & OF & $\mathbf{T}$ & B & FU & PU & NU & LNU & $\mathbf{S}$ & GAP \\
\hline 01 & SSSS & 152.73 & 547.50 & 29.80 & 29.70 & 0.10 & 0.10 & 0.30 & 0.00 & $0.41 \%$ \\
\hline 02 & SSSM & 274.41 & 186.55 & 53.70 & 53.70 & 0.00 & 0.00 & 0.00 & 0.00 & $0.03 \%$ \\
\hline 03 & SSSL & 8702.17 & 7.32 & 85.90 & 85.90 & 0.00 & 0.00 & 0.00 & 17.20 & $0.00 \%$ \\
\hline 04 & SSMS & 97.67 & 830.45 & 16.90 & 14.30 & 2.60 & 0.00 & 0.00 & 0.00 & $1.52 \%$ \\
\hline 05 & SSMM & 205.91 & 191.18 & 40.20 & 40.10 & 0.10 & 0.10 & 1.10 & 0.00 & $0.25 \%$ \\
\hline 06 & SSML & 411.87 & 11.48 & 80.60 & 80.60 & 0.00 & 0.00 & 0.00 & 0.00 & $0.00 \%$ \\
\hline 07 & SSLS & 116.35 & 1462.04 & 20.30 & 17.40 & 2.90 & 0.00 & 0.00 & 0.00 & $2.97 \%$ \\
\hline 08 & SSLM & 158.48 & 1800.00 & 29.80 & 28.50 & 1.30 & 0.50 & 10.90 & 0.00 & $5.93 \%$ \\
\hline 09 & SSLL & 388.30 & 373.48 & 75.90 & 75.80 & 0.10 & 0.10 & 0.40 & 0.00 & $0.13 \%$ \\
\hline 10 & SMSS & 296.38 & 190.40 & 58.00 & 58.00 & 0.00 & 0.00 & 0.00 & 0.00 & $0.05 \%$ \\
\hline 11 & SMSM & 549.33 & 372.87 & 107.50 & 107.50 & 0.00 & 0.00 & 0.00 & 0.00 & $0.02 \%$ \\
\hline 12 & SMSL & 19612.76 & 11.40 & 171.50 & 171.50 & 0.00 & 0.00 & 0.00 & 39.00 & $0.00 \%$ \\
\hline 13 & SMMS & 173.47 & 1800.00 & 32.40 & 30.60 & 1.80 & 0.30 & 1.50 & 0.00 & $5.73 \%$ \\
\hline 14 & SMMM & 392.96 & 195.72 & 76.90 & 76.90 & 0.00 & 0.00 & 0.00 & 0.00 & $0.08 \%$ \\
\hline 15 & SMML & 818.11 & 199.60 & 160.10 & 160.10 & 0.00 & 0.00 & 0.00 & 0.00 & $0.00 \%$ \\
\hline 16 & SMLS & 211.13 & 1632.34 & 39.70 & 37.80 & 1.90 & 0.00 & 0.00 & 0.00 & $3.85 \%$ \\
\hline 17 & SMLM & 305.56 & 1452.71 & 59.20 & 58.50 & 0.70 & 0.00 & 0.00 & 0.00 & $2.15 \%$ \\
\hline 18 & SMLL & 772.63 & 217.67 & 151.20 & 151.20 & 0.00 & 0.00 & 0.00 & 0.00 & $0.01 \%$ \\
\hline 19 & SLSS & 436.91 & 200.11 & 85.50 & 85.50 & 0.00 & 0.00 & 0.00 & 0.00 & $0.01 \%$ \\
\hline 20 & SLSM & 817.60 & 28.38 & 160.00 & 160.00 & 0.00 & 0.00 & 0.00 & 0.00 & $0.00 \%$ \\
\hline 21 & SLSL & 33741.62 & 562.03 & 257.00 & 257.00 & 0.00 & 0.00 & 0.00 & 67.50 & $0.07 \%$ \\
\hline 22 & SLMS & 250.28 & 1800.00 & 48.00 & 46.90 & 1.10 & 0.10 & 4.30 & 0.00 & $2.65 \%$ \\
\hline 23 & SLMM & 602.98 & 31.45 & 118.00 & 118.00 & 0.00 & 0.00 & 0.00 & 0.00 & $0.00 \%$ \\
\hline 24 & SLML & 1225.89 & 416.55 & 239.90 & 239.90 & 0.00 & 0.00 & 0.00 & 0.00 & $0.01 \%$ \\
\hline 25 & SLLS & 305.25 & 1534.72 & 58.80 & 57.70 & 1.10 & 0.00 & 0.00 & 0.00 & $1.89 \%$ \\
\hline 26 & SLLM & 450.46 & 1800.00 & 87.30 & 86.30 & 1.00 & 0.10 & 0.20 & 0.00 & $1.16 \%$ \\
\hline 27 & SLLL & 1152.82 & 213.84 & 225.60 & 225.60 & 0.00 & 0.00 & 0.00 & 0.00 & $0.00 \%$ \\
\hline 28 & DSSS & 152.64 & 1439.39 & 29.70 & 29.50 & 0.20 & 0.10 & 0.10 & 0.00 & $2.34 \%$ \\
\hline 29 & DSSM & 272.87 & 303.31 & 53.40 & 53.40 & 0.00 & 0.00 & 0.00 & 0.00 & $0.10 \%$ \\
\hline 30 & DSSL & 5434.22 & 144.88 & 85.60 & 85.50 & 0.10 & 0.10 & 0.10 & 10.40 & $0.00 \%$ \\
\hline 31 & DSMS & 110.84 & 1800.00 & 17.60 & 12.90 & 4.70 & 0.10 & 9.20 & 0.00 & $14.00 \%$ \\
\hline 32 & DSMM & 204.19 & 1252.65 & 39.70 & 39.40 & 0.30 & 0.30 & 0.40 & 0.00 & $1.35 \%$ \\
\hline 33 & DSML & 413.33 & 529.90 & 80.80 & 80.70 & 0.10 & 0.10 & 0.10 & 0.00 & $0.25 \%$ \\
\hline 34 & DSLS & 134.17 & 1800.00 & 22.00 & 17.00 & 5.00 & 0.00 & 0.00 & 0.00 & $14.35 \%$ \\
\hline 35 & DSLM & 170.53 & 1645.78 & 31.30 & 28.90 & 2.40 & 0.40 & 3.00 & 0.00 & $10.78 \%$ \\
\hline 36 & DSLL & 391.67 & 1172.98 & 76.30 & 75.90 & 0.40 & 0.40 & 0.70 & 0.00 & $0.71 \%$ \\
\hline 37 & DMSS & 293.75 & 1357.39 & 57.40 & 57.30 & 0.10 & 0.00 & 0.00 & 0.00 & $1.53 \%$ \\
\hline 38 & DMSM & 535.53 & 1134.51 & 104.80 & 104.80 & 0.00 & 0.00 & 0.00 & 0.00 & $0.52 \%$ \\
\hline 39 & DMSL & 21248.21 & 515.41 & 171.90 & 171.90 & 0.00 & 0.00 & 0.00 & 42.40 & $0.09 \%$ \\
\hline 40 & DMMS & 185.92 & 1800.00 & 33.30 & 29.70 & 3.60 & 0.10 & 1.90 & 0.00 & $10.87 \%$ \\
\hline 41 & DMMM & 406.68 & 1312.54 & 79.50 & 79.40 & 0.10 & 0.00 & 0.00 & 0.00 & $0.94 \%$ \\
\hline 42 & DMML & 822.71 & 809.41 & 161.00 & 161.00 & 0.00 & 0.00 & 0.00 & 0.00 & $0.16 \%$ \\
\hline 43 & DMLS & 220.71 & 1800.00 & 39.70 & 35.60 & 4.10 & 0.10 & 0.10 & 0.00 & $8.38 \%$ \\
\hline 44 & DMLM & 313.25 & 1800.00 & 58.90 & 56.10 & 2.80 & 0.40 & 1.90 & 0.00 & $5.87 \%$ \\
\hline 45 & DMLL & 773.08 & 965.35 & 151.20 & 151.10 & 0.10 & 0.10 & 0.20 & 0.00 & $0.29 \%$ \\
\hline 46 & DLSS & 451.14 & 936.51 & 88.00 & 88.00 & 0.10 & 0.00 & 0.00 & 0.00 & $0.66 \%$ \\
\hline 47 & DLSM & 818.11 & 1629.38 & 160.10 & 160.10 & 0.00 & 0.00 & 0.00 & 0.00 & $0.40 \%$ \\
\hline 48 & DLSL & 33888.81 & 972.64 & 257.60 & 257.60 & 0.00 & 0.00 & 0.00 & 67.80 & $0.14 \%$ \\
\hline 49 & DLMS & 261.28 & 1800.00 & 48.10 & 44.60 & 3.50 & 0.10 & 5.30 & 0.00 & $7.44 \%$ \\
\hline 50 & DLMM & 613.56 & 1614.38 & 119.90 & 119.70 & 0.20 & 0.00 & 0.00 & 0.00 & $0.94 \%$ \\
\hline 51 & DLML & 1227.86 & 1356.66 & 240.20 & 240.10 & 0.10 & 0.10 & 0.10 & 0.00 & $0.12 \%$ \\
\hline 52 & DLLS & 319.52 & 1672.56 & 60.40 & 57.90 & 2.50 & 0.00 & 0.00 & 0.00 & $4.09 \%$ \\
\hline 53 & DLLM & 462.86 & 1800.00 & 89.00 & 87.00 & 2.00 & 0.20 & 0.70 & 0.00 & $3.59 \%$ \\
\hline 54 & DLLL & 1155.74 & 1637.42 & 226.00 & 226.00 & 0.00 & 0.10 & 0.10 & 0.00 & $0.32 \%$ \\
\hline
\end{tabular}

Caption: OF: Objective Function; T: Processing time in seconds; B: Average number of reels used; FU: Average number of reels that are fully used; PU: Average number of reels that are partially used; NU: Average number of reels with unusable leftovers; LNU: Length of unusable leftovers; S: Average number of stoppages; GAP: Average value of the relative GAPs 
The general analysis in Table 3 shows that the model meets the main objectives proposed.

Partial Consumption $\left(\beta_{2}\right)$ : In an ideal situation for each schedule, the plan should allow a maximum of one partial use per reel holder, i.e. at most three partial uses for single wall schedules or five partial uses for double wall schedules. The results in Table 3 shows that, considering the whole set of 540 instances, only $44(8.14 \%)$ instances have three partial uses and only $23(4.25 \%)$ have five partial uses, with almost all reels being fully used.

Unusable leftovers $\left(\beta_{3}\right)$ : In the CCPC visited in this study, around 30 tonnes of unusable leftovers were generated and 200 schedules were processed on average per month. About 12 reels are used per schedule, providing a total monthly average of 2400 reels and an average loss of $12.5 \mathrm{~kg} / \mathrm{reel}$. The production is planned by the CCPC without the aid of any planning tools. Chantrapornchai \& Sathapanawat (2012) present a case study in which the loss is $5.38 \mathrm{~kg} / \mathrm{reel}$ using a computational tool. In computational testing of the proposed model in the present study, 51,531 reels were used in the 54 classes analyzed which generated a loss of $401 \mathrm{~kg}$ i.e. the loss was $0.007 \mathrm{~kg} / \mathrm{reel}$, showing that the results from the model can be better than current practice in the CCPC visited and those reported by Chantrapornchai \& Sathapanawat (2012).

Use of reels $\left(\beta_{1}\right)$ and Stoppages $\left(\beta_{4}\right)$ : In relation to the number of reels used and the number of stoppages, direct analysis is not possible. In practice, stoppages are avoided by using reels with a lot of paper that allow many changes, which obviously reduces the total number of reels and the number of stoppages. This strategy generates many reusable reels during the production process, leading to the accumulation of these shorter length, reusable reels in stock, which are then difficult to manage and to allocate for reuse.

As shown in Table 3, the CPLEX solver did not prove optimality of 274 instances at timeout. Overall, in $93.33 \%$ of the instances analyzed, no leftover waste is generated, which implies a reduction in recycling costs. In relation to partial usage, for 3-layer schedules, there are, at most, three partially-used reels and for double-layer schedules, at most five, the ideal situation in practical terms. In $88.89 \%$ of instances there are no stoppages. Optimal solutions are obtained in $49.25 \%$ of the instances.

According to Table 4 the 110 instances for which CPLEX did not prove optimality are for the classes SSLM, SMMS, SLMS, SLLM, DSMS, DSLS, DMMS, DMLS, DMLM, DLMS and DLLM with all ten instances in these classes not being proven optimal (note in Table 4 that the average computational time of these classes exceeds the time limit of 1800 seconds). Of these 11 classes, it is worth noting that $63.63 \%$ are double-walled packaging, $100 \%$ of these instances focus on cases when the lengths of paper on the reels in stock are M or L and $100 \%$ of the instances have the length of the schedule as size S and M, $63.63 \%$ being of type S. Also according to Table 3, the general average for the 54 classes of instances produced an objective value of 2740.03, in an average computational time of 983.82 seconds and with an average GAP 
of $2.21 \%$, which is quite reasonable for a real problem. On average, 95.43 reels per instance were used, of which 94.56 on average were fully used and 0.87 partially used and, of these partially used reels, 0.07, on average, generated unusable leftovers with an average length of $0.79 \mathrm{~m}$. Finally, the average number of stoppages per instance was 4.52 .

\subsection{The influence of each parameter on the computational analysis}

In this section, there is an analysis to understand the influence of each parameter independently on the computational results. The following results were obtained based on the results in Table 4.

Table 5 - Comparison of schedule layers.

\begin{tabular}{lccccccccc}
\hline & OF & T & B & FU & PU & NU & LNU & S & GAP \\
\hline Single Wall & 2689.78 & 669.86 & 95.17 & 94.63 & 0.54 & 0.05 & 0.69 & 4.58 & $1.07 \%$ \\
Double Wall & 2640.12 & 1297.78 & 95.68 & 94.46 & 1.21 & 0.10 & 0.89 & 4.47 & $3.34 \%$ \\
\hline
\end{tabular}

Table 5 shows an analysis of the number of schedule layers and it can be seen that, in terms of the objective function, a slight reduction is seen for double-walled packaging, given by the reduction in the average number of stoppages. Note that the length of the schedule is given according to the length of paper on the reels in stock. The same percentage is used for both types of corrugated packaging, therefore, in double-wall cardboard, the number of changes will be smaller, which reduces the number of stoppages. As for processing time and GAP, there is an increase for the double walled packaging, which is expected since two extra layers must be processed increasing the number of model variables. The average number of reels used as well as the average number of partially-used reels and the length of unusable leftovers was slightly higher for the double-walled cardboard, which is expected due to the increase in the number of layers.

Table 6 shows that increasing the number of reels in stock increases the value of the objective function and the processing time. The increase in processing time is due to the increase in the number of variables while the objective function increases because the length of the schedule is defined according to the stock. Therefore, larger inventories generate larger schedules for which higher values are expected for the objective function. This fact also explains the increase in the number of stoppages. The results indicate that the GAP decreases as stock size increases because, although there are more reels to match, there are better reels in stock which makes it easy to find good solutions. As full use of reels is a priority, the increase in the number of reels available is accompanied by an increase in the number of fully used reels and a reduction in partial use. This reduction in partial consumption is accompanied by a reduction in the amount of unusable leftovers which necessarily only results from partial use.

In Table 7, the paper length on reels in stock is analyzed. The value of the objective function decreases as paper lengths on reels increases, which can be explained by the reduction in the number of stoppages. Conversely, processing time and GAP increase indicating that the problem becomes more difficult to solve. In relation to the amount of instances with unusable leftovers, 
Table 6 - Comparison of the number of reels in stock.

\begin{tabular}{lccccccccc}
\hline & OF & T & B & FU & PU & NU & LNU & S & GAP \\
\hline Small Stock & 988.46 & 861.62 & 48.31 & 47.18 & 1.13 & 0.13 & 1.46 & 1.53 & $3.06 \%$ \\
Medium Stock & 2662.90 & 976.99 & 95.23 & 94.39 & 0.84 & 0.06 & 0.31 & 4.52 & $2.25 \%$ \\
Large Stock & 4343.48 & 1112.84 & 142.74 & 142.07 & 0.67 & 0.04 & 0.59 & 7.52 & $1.31 \%$ \\
\hline
\end{tabular}

the longer the paper lengths on reels in stock, the more instances with unusable leftovers are generated. This is because there is a reduction in the percentage of instances with fully used reels only and an increase in the percentage of instances with one or more partially used reels and these are the instances in which unusable leftovers arise.

Table 7 - Comparison of paper lengths on reels in stock.

\begin{tabular}{lccccccccc}
\hline & OF & T & B & FU & PU & NU & LNU & S & GAP \\
\hline Short Profile & 7093.29 & 585.55 & 112.09 & 112.06 & 0.03 & 0.02 & 0.03 & 13.57 & $0.35 \%$ \\
Medium Profile & 468.08 & 987.59 & 90.73 & 89.72 & 1.01 & 0.07 & 1.33 & 0.00 & $2.57 \%$ \\
Large Profile & 433.47 & 1378.30 & 83.46 & 81.87 & 1.59 & 0.13 & 1.01 & 0.00 & $3.69 \%$ \\
\hline
\end{tabular}

Finally, analysis of the schedule length in Table 8 shows that the value of the objective function increases with the length of the schedule. As expected, larger schedules naturally consume more reels. The significant increase in the number of stoppages when the schedule is large must be highlighted. Processing time and GAP decrease as the schedule length increases because large schedules consume by definition $95 \%$ of the stock, i.e. practically all the reels in stock are used, simplifying the analysis. Regarding the number of instances with unusable leftovers, small and large schedules are similar. The medium-sized schedules are those with the highest number of unusable leftover reels. The length of the unusable leftovers is clearly inversely proportional to the length of the schedule. By analyzing the averages of fully used reels, these values increase when the schedule length increases and there is a consequent reduction in partial use.

Table 8 - Comparison of schedule lengths.

\begin{tabular}{lccccccccc}
\hline & OF & T & B & FU & PU & NU & LNU & S & GAP \\
\hline Small Schedule & 231.67 & 1357.78 & 43.66 & 41.69 & 1.96 & 0.06 & 1.26 & 0.00 & $4.60 \%$ \\
Medium Schedule & 419.74 & 1031.55 & 81.61 & 80.99 & 0.62 & 0.11 & 1.01 & 0.00 & $1.89 \%$ \\
Large Schedule & 7343.43 & 562.11 & 161.02 & 160.96 & 0.06 & 0.06 & 0.09 & 13.57 & $0.13 \%$ \\
\hline
\end{tabular}




\section{CONCLUSIONS AND FUTURE WORK}

The problem of reel allocation to the corrugator is relevant in reducing costs in the production process of corrugated packaging companies. Several objectives are addressed in this planning, such as minimizing unusable leftovers, minimizing the number of partially-used reels and the minimization of the number of machine stoppages. In this paper, we propose a mathematical model to the reel allocation problem to assist in the choice of the set of reels that presents minimum costs to reach the objectives. Computational tests were performed with instances based on real data and the results indicate that the proposed mathematical model is consistent with the reality of the studied company and brings interesting insights that can be used in the decision-making.

In this study, the reel allocation problem was individually dealt with each production schedule. An interesting aspect to be investigated is to look at the definition of a sequence of several schedules to be produced. The introduction of this specificity to the approach could show further reductions in costs, remembering that, in the current study, the first two reels of each reel holder set are pre-installed. Another important issue to be considered as future research is the problem of determining the sequence in which the reels will be installed on the reel holders. For example, one can integrate this problem with the current mathematical model. Studying ways to avoid aging of the reels in stock and the relation of the reel allocation problem to the forklift displacement problem are also interesting topics for future research.

\section{Acknowledgements}

This research was funded by the Conselho Nacional de Desenvolvimento Científico e Tecnológico - CNPq, Coordenação de Aperfeiçoamento de Pessoal de Nível Superior - CAPES and the Fundação de Amparo a Pesquisa do Estado de São Paulo - FAPESP (process n. 2013/07375-0 and 2016/01860-1).

\section{References}

[1] Arenales MN, Cherri AC, Nascimento DN \& Vianna ACG. 2015. A new mathematical model for the cutting stock/leftover problem. Pesquisa Operacional, 35: 509 522.

[2] BecCeneri JC, YAnASSE HH \& Soma NY. 2004. A method for solving the minimization of the maximum number of open stacks problem within a cutting process. Computers \& Operations Research, 31(14): 2315-2332.

[3] Bolat A. 2000. An extended scheduling model for producing corrugated boxes. International Journal of Production Research, 38(7): 1579-1599.

[4] Bookbinder JH \& Higginson JK. 1986. Customer service vs trim waste in corrugated box manufacture. Journal of the Operational Research Society, 37(11): 1061-1071. 
[5] Chantrapornchai C \& Sathapanawat T. 2010. Scheduling and planning software for corrugation process. In: Signal Processing and Multimedia. pp. 260-266. Springer.

[6] Chantrapornchai C \& Sathapanawat T. 2011. Heuristic for paper roll selection for corrugation process simulation. In: $U$-and E-Service, Science and Technology. pp. 153-159. Springer.

[7] Chantrapornchai C \& Sathapanawat T. 2012. Corrugation Software Development and Deployment: A Case Study. International Journal of u-and e-Service, Science and Technology, 5(1): 1-24.

[8] Cherri AC, Arenales Mn, Yanasse HH, Poldi KC \& Vianna ACG. 2014. The one-dimensional cutting stock problem with usable leftovers - A survey. European Journal of Operational Research, 236(2): 395 - 402.

[9] DARley V, SANDERS D \& VON TESSin P. 2004. An agent-based model of a corrugatedbox factory: the trade-off between finished goods stock and on-time-in-full delivery. In: Proceedings of the Fifth Workshop on Agent-Based Simulation.

[10] Haessler RW \& TAlbot FB. 1983. A 0-1 model for solving the corrugator trim problem. Management science, 29(2): 200-209.

[11] Jaramillo JD, Correa FJ \& Jaramillo R. 2009. Desarrollo de un método basado en algoritmos genéticos y programación lineal para la solución de un problema de corte unidimensional. Enviado a revisión a la Revista de Ingeniería de la Universidad Eafit, .

[12] LiNS S. 1989. Traversing trees and scheduling tasks for duplex corrugator machines. Pesquisa Operacional, 9(1): 40-54.

[13] Matsumoto K, Miwa H \& Ibaraki T. 2009. Scheduling of corrugated paper production. European Journal of Operational Research, 192(3): 782-792.

[14] Pegels CC. 1967. A comparison of scheduling models for corrugator production. Journal of Industrial Engineering, 18(8): 466.

[15] Poltroniere SC, Poldi KC, Toledo FMB \& Arenales MN. 2008. A coupling cutting stock-lot sizing problem in the paper industry. Annals of Operations Research, 157(1): 91-104.

[16] Rodríguez MA \& VecChietti A. 2007. An efficient model implementation to solve a real-world cutting stock problem for a corrugated board boxes mill. Computer Aided Chemical Engineering, 24: 601.

[17] Rodriguez MA \& Vecchietti A. 2013. Integrated Planning and Scheduling with Due Dates in the Corrugated Board Boxes Industry. Industrial \& Engineering Chemistry Research, 52(2): 847-860. Available at: https://doi.org/10.1021/ie302109j. 
[18] RodRÍGUEZ TFT. 2009. Una combinación de un algoritmo voraz con algoritmos genéticos para optimizar la producción de cartón ondulado. Revista de métodos cuantitativos para la economía y la empresa, 8: 71-86.

[19] Santos MO \& AlmadA-Lobo B. 2012. Integrated pulp and paper mill planning and scheduling. Computers \& Industrial Engineering, 63(1): 1-12.

[20] SAVSAR M \& Cogun C. 1994. Analysis and modelling of a production line in a corrugated box factory. The International Journal of Production Research, 32(7): 1571-1589.

[21] Velasquez A, Bellini G, Paternina-Arboleda CD et Al. 2007. A multiobjective approach based on soft computing techniques for production scheduling in Corrugator manufacturing plants. Ingeniería y Desarrollo, 21.

\section{How to cite}

Ferreira M, Camargo VCb \& Araujo SA. 2020. The Reel Allocation Problem of a Corrugated Packaging Company. Pesquisa Operacional, 40: e229708. doi: 10.1590/0101-7438.2020.040.00229708. 


\section{APPENDIX}

\section{Sequencing problem: a procedure that simulates the practice}

The solution of the mathematical model (1)-(14) gives how much paper will be consumed from each reel and to which reel holders they will be assigned but it does not define the usage sequence. For each reel holder assembly, there are four possibilities in this reel sequencing problem:

- If all selected reels allow for at least one change, regardless of sequencing, there will be no stoppages.

- If none of the selected reels allow changes regardless of sequencing, the number of stoppages equals $m-2$.

- If, for a given reel holder assembly only two reels are allocated, just putting them on pins 1 and 2 solves the problem.

- If the number of reels selected for a particular reel holder is greater than 3 , use the procedure described as follows.

\section{Reel sequencing procedure:}

In this procedure, reels with partial and total use will be treated the same way. For each reel holder $j$, do:

1. Sort the $\bar{m}=\sum_{i=1}^{m} Y_{i j}$ reels selected for reel holder $j$ in decreasing order of paper length to be used from each one $\left(X_{i j}\right)$, obtaining the set: $\left\{a_{1}, a_{2}, \ldots, a_{\bar{m}}\right\}$

2. Calculate the number of changes allowed by reel $a_{1}$. Just calculate $\left\lfloor X_{a_{1}, j} / p\right\rfloor$, denote this value as $k_{1}$, assuming $k_{1} \geq 1$. If $k_{1}=0$, none of the $\bar{m}$ reels allow for changes, and in this case, regardless of sequencing, the number of stoppages is equal to $\bar{m}-2$, by Definition 4.1 .

3. Reel $a_{1}$ will be placed on Pin 1 of this reel holder and, on Pin 2, place reels in positions: $\bar{m}, \bar{m}-1, \ldots, \bar{m}-\left(k_{1}-1\right)$, in this order. Pin 2 will be the first active pin until reel from position $\bar{m}$ is used to length $X_{\bar{m}, j}$. Then pin 1 becomes active using reel $a_{1}$, while reel in position $\bar{m}-1$ is installed on pin 2 . Immediately after the change, pin 1 becomes inactive, pin 2 becomes active and the reel in position $\bar{m}-1$ is consumed to length $X_{\bar{m}-1, j}$. This must then be repeated until the reel in position $\bar{m}-\left(k_{1}-1\right)$ has been used. The sequencing ends when $\bar{m}-\left(k_{1}-1\right)=2$. Otherwise, the remaining paper from reel $a_{1}$ should be consumed while reel $a_{2}$ is installed. Go to step 4 .

4. Repeat the 2nd and 3rd steps for reel $a_{2}$ disregarding reels already used along with reel $a_{1}$. If the reels run out, stop. If not, repeat the 2 nd and 3 rd steps for reel $a_{3}$ and so on. 